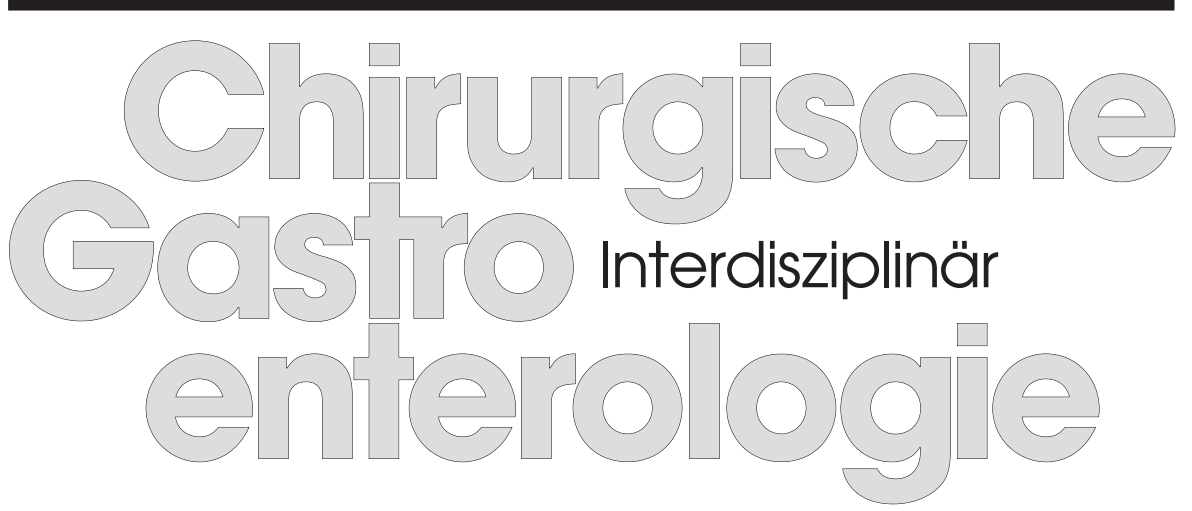

Hauptthema

R.A. Weiner, Frankfurt/M. (Hrsg.)

\title{
Laparoskopische Adipositas-Chirurgie
}

\author{
Eingeladene Beiträge \\ Interdisziplinäres Gespräch
}

Weitere Rubriken

Originalarbeit

Briefe an die Herausgeber

Buchbesprechungen

Tagungen und Kongresse 


\section{Band 19, Heft 1, März 2003}

Gegründet 1984 als «Chirurgische

Gastroenterologie mit interdisziplinären

Gesprächen» durch A. Akovbiantz, H. Denk,

K.-J. Paquet und C.E. Zöckler.

\section{Hauptschriftleitung}

R. Bittner, Stuttgart

\section{Schriftleitung Viszeralchirurgie}

E. Klar, Heidelberg

\section{Schriftleitung Gastroenterologie}

J. Mössner, Leipzig

\section{Assistenz Schriftleitung}

C.-G. Schmedt, Stuttgart

\section{Fachschriftleitung}

Viszeralchirurgie

J. R. Izbicki, Hamburg

H. Lippert, Magdeburg

H. Säuberli, Baden

R. Schiessel, Wien

Gastroenterologie

H. Bosseckert, Jena

Minimal invasive Chirurgie

H.-P. Bruch, Lübeck

I. Gastinger, Cottbus

Transplantation

C. E. Broelsch, Essen

J. Hauss, Leipzig

\section{Berufspolitik}

K.-J. Paquet, Bad Kissingen

B. Ulrich, Düsseldorf

Chirurgische Onkologie

M.W. Büchler, Heidelberg

K. Glaser, Wien

\section{Wissenschaftlicher Beirat}

\section{Viszeralchirurgie}

J. Lange, St. Gallen

H.B. Reith, Würzburg

H.-D. Saeger, Dresden

M. Schilling, Homburg/Saar

\section{Gastroenterologie}

T. Andus, Stuttgart

W. E. Fleig, Halle

M. Fried, Zürich

E. G. Hahn, Erlangen-Nürnberg

J. Schölmerich, Regensburg

Minimal invasive Chirurgie

F. Köckerling, Hannover

R. Schlumpf, Aarau

K. Schönleben, Ludwigshafen

G. Szinicz, Bregenz

W. Wayand, Linz

Berufspolitik

M. Betzler, Essen

K. Junghanns, Ludwigsburg

Chirurgische Onkologie

W. Hohenberger, Erlangen-Nürnberg

\section{Onkologie}

S. von Hegewisch-Becker, Hamburg

R. Porschen, Bremen

\section{Internationaler Beirat}

J. Gallinger, Moskau

J.M. Henderson, Cleveland, $\mathrm{OH}$

I. Ihse, Lund

C. W. Imrie, Glasgow

S. L. Jensen, Arhus

K. Maruyama, Tokyo

W. C. Meyers, Worcester, MA
Endoskopie

S. Liebe, Rostock

P. Malfertheiner, Magdeburg

J.F. Riemann, Ludwigshafen

Perioperatives Management

K.-W. Jauch, Regensburg

H. Wiedeck, Ulm

D. H. Wittmann, Venice, FL

K. Hell, Basel

Radiologie

M. Langer, Freiburg i. Br.

Pathologie

P. Hermanek, Erlangen-Nürnberg

C. Wittekind, Leipzig

Klinische Molekularbiologie

H. Friess, Heidelberg

Weiterbildung

J. Jähne, Hannover

Innovationen, Aktuelle Trends,

Kongressberichte

J. Schmidt, Heidelberg

W. G. Zoller, Stuttgart

Buchbesprechungen

K.-J. Paquet, Hannover

S. Karger $\cdot$ Medical and Scientific Publishers $\cdot$ Basel $\cdot$ Freiburg $\cdot$ Paris $\cdot$ London $\cdot$ New York $\cdot$ New Delhi $\cdot$ Bangkok $\cdot$ Singapore $\cdot$ Tokyo $\cdot$ Sydney

Die Zeitschrift erscheint vierteljährlich; pro Jahr erscheint 1 Band zu je 4 Heften. Bezugspreise für Jahrgang 19, 2003: Print-Abonnement EUR 160,-, Online-Abonnement EUR 160,-, Kombi-Abonnement Print/Online EUR 188,-, einschließlich MwSt., zuzüglich Postgebühren. Der Abonnementpreis ist im Voraus zahlbar. Das Abonnement der Zeitschrift läuft weiter, wenn es nicht spätestens 4 Wochen vor Abschluss eines Bandes abbestellt wird. Abonnementbestellungen können bei jeder Buchhandlung oder direkt beim Verlag aufgegeben werden:

\section{Deutschland:}

S. Karger $\mathrm{GmbH}$

Lörracher Str. 16a

D-79115 Freiburg

Tel. +49761452070

Fax +497614520714

E-mail Information@Karger.de

\section{Anzeigen: S. Karger}

Verlag für Medizin und Naturwissenschaften $\mathrm{GmbH}$ Lörracher Str.16a, D-79115 Freiburg, Tel. +49 761452070. Gültig ist die Preisliste Nr. 10 vom 1. Januar 2003.

Für den Inhalt ausserhalb des redaktionellen Teiles (insbesondere Anzeigen, Industrieinformationen, Pressezitate und Kongressinformationen) übernehmen Schriftleitung, Beirat und Verlag keine Gewähr.

Eine Markenbezeichnung kann warenzeichenrechtlich geschützt sein, auch wenn bei ihrer Verwendung in dieser Zeitschrift das Zeichen ${ }^{\circledR}$ oder ein anderer Hinweis auf etwa bestehende Schutzrechte fehlen sollte. Für Satzfehler, insbesondere bei Dosierungsangaben, wird keine Gewähr übernommen.

Die Zeitschrift sowie alle in ihr enthaltenen einzelnen Beiträge und Abbildungen sind urheberrechtlich geschützt. Jede Verwertung, die nicht ausdrücklich vom Urheberrechtsgesetz zu- gelassen ist, bedarf der vorherigen Zustimmung des Verlags. Das gilt insbesondere für Vervielfältigungen, Bearbeitungen, Übersetzungen, Mikroverfilmungen und die Einspeicherung und Verarbeitung in elektronischen Systemen.

(c) Copyright 2002 by S. Karger

Verlag für Medizin und Naturwissenschaften GmbH Lörracher Str. 16a, D-79115 Freiburg

Verlagsleitung und presserechtlich verantwortlich: Sibylle Hopf

Anzeigenleitung: Susanne Meister

Produktionsleitung: Georg Brunner

Gesamtherstellung: KONKORDIA GmbH, Bühl Das Medienunternehmen

ISBN 3-8055-7562-9

\section{KARGER}

Fax +497614520714 E-mail Information@Karger.de www.karger.com
(C) 2003 S. Karger GmbH, Freiburg

Home Page der Zeitschrift:

www.karger.com/cga
Bibliographische Dienste: EMBASE / Excerpta Medica Reference Update 
Band 19, Heft 1, März 2003

Editorial

6 Die operative Behandlung der Adipositas Weiner, R.A. (Frankfurt/M.)

Hauptthema

8 Ist Adipositas eine Krankheit?

Hauner, H. (Düsseldorf)

12 Psychosomatik der Adipositas Schüler-Schneider, A. (Frankfurt/M.)

22 Adipositas-Chirurgie: Historie - Gegenwart - Zukunft Husemann, B.J. (Düsseldorf)

30 Das laparoskopisch implantierte steuerbare Magenband Beobachtungen und Entwicklungen

Blanco-Engert, R.; Matkowitz, R. (Frankfurt/M.); Weiner, S. (Würzburg); Diaz Maag, R. (Merida); Weiner, R. (Frankfurt/M.)

35 Indikation und Technik beim verstellbaren Magenband Müller, W.; Beller, S.; Szinicz, G. (Bregenz)

38 Behandlung der Refluxösophagitis bei extremer Adipositas - ein Fallbericht

Reijnen, N.P.M.; Shahbazian-Bscheidl, Z. (Leer)

41 Die laparoskopische vertikale Magenseparation ein neuer Zugangsweg für ein Standardverfahren Bröhl, F. (Osnabrück)

46 Laparoskopischer Roux-en-Y-Magen-Bypass - Technik und Komplikationen

Weiner, R.A.; Blanco-Engert, R.; Winterberg, U. (Frankfurt/M.)

54 Laparoskopischer Duodenal-Switch - Technik und erste Erfahrungen

Baltasar, A.; Bou, R.; Miro, J.; Bengochea, M.; Serra, C.; Pérez, N. (Alcoy, Alicante)

57 «anded» oder funktioneller Magen-Bypass? Weiner, R.A. (Frankfurt/M.)

62 Redo-Chirurgie: Umwandlung von einem bariatrischen Verfahren in ein anderes Weiner, R.A. (Frankfurt/M.)

70 Lebensqualität nach bariatrischen Eingriffen - ein Überblick Weiner, S. (Würzburg); Weiner, R.A.; Pornhoff, I. (Frankfurt/M.)
Vol. 19, Issue 1, March 2003

Editorial

6 Surgical Treatment of Obesity Weiner, R.A. (Frankfurt/M.)

Main Topic

8 Is Obesity a Disease?

Hauner, H. (Düsseldorf)

12 Psychosomatics of Obesity Schüler-Schneider, A. (Frankfurt/M.)

22 Obesity Surgery: History - Present - Future Husemann, B.J. (Düsseldorf)

30 Laparoscopically Implanted Adjustable Gastric Banding - Observations and Evolution

Blanco-Engert, R.; Matkowitz, R. (Frankfurt/M.); Weiner, S. (Würzburg); Diaz Maag, R. (Merida); Weiner, R. (Frankfurt/M.)

35 Indication and Technique of the Adjustable Gastric Band Müller, W.; Beller, S.; Szinicz, G. (Bregenz)

38 Treatment of GERD in Patients with Morbid Obesity a Case Report

Reijnen, N.P.M.; Shahbazian-Bscheidl, Z. (Leer)

41 Laparoscopic Vertical Gastroplasty - a New Approach for a Standard Procedure Bröhl, F. (Osnabrück)

46 Laparoscopic Roux-en-Y-Gastric Bypass - Technique and Complications

Weiner, R.A.; Blanco-Engert, R.; Winterberg, U. (Frankfurt/M.)

54 Laparoscopic Duodenal Switch - Technique and Initial Experience

Baltasar, A.; Bou, R.; Miro, J.; Bengochea, M.; Serra, C.; Pérez, N. (Alcoy, Alicante)

57 Banded or Functional Gastric Bypass?

Weiner, R.A. (Frankfurt/M.)

62 Redo Surgery: Transformation of a Bariatric Treatment in Another

Weiner, R.A. (Frankfurt/M.)

70 Quality of Life after Bariatric Treatment - an Overview Weiner, S. (Würzburg); Weiner, R.A.; Pornhoff, I. (Frankfurt/M.)

\begin{tabular}{ll}
\hline KARGER & ( 2003 S. Karger GmbH, Freiburg \\
Fax +497614520714 & Accessible online at: \\
$\begin{array}{l}\text { E-mail Information@Karger.de } \\
\text { www.karger.com }\end{array}$ & www.karger.com/cga
\end{tabular}


Band 19, Heft 1, März 2003

Interdisziplinäre Diskussion

76 Operative Therapie der Adipositas

Husemann, B. (Düsseldorf) (Diskussionsleiter)

Originalarbeiten

81 Die vaskuläre Anatomie der vorderen Bauchwand: Ein Beitrag zur Vermeidung von Gefäßverletzungen bei der laparoskopischen Chirurgie

Balzer, K.M. (Düsseldorf); Witte, H. (Jena); Faustmann, P. (Bochum); Kozianka, J.; Waleczek, H. (Herne)

86 Selbstentfaltende Metall-Stents zur Behandlung der Obstruktion des Kolons

Dell'Abate, P.; Del Rio, P.; Colla, G.; Soliani, P.; Arcuri, M.F.; Ziegler, S.; Sianesi, M. (Parma)

89 Palliative Chemotherapie bei kolorektalen Karzinomen Ergebnisse einer Umfrage

Manekeller, S.; Lauschke, H.; Tolba, R.; Minor, T. (Bonn); Gallkowski, U. (Neuwied); Hirner, A. (Bonn)

Brief an die Herausgeber

96 Verbesserte Früherkennung von Darmkrebs Classen, M. (Gastro-Liga); Riemann, J.F. (Lebensblicke); Schmiegel, W.-H. (DGVS)

99 Buchbesprechungen

101 Tagungen und Kongresse

2 Impressum

103 Hinweise für Autoren
Vol. 19, Issue 1, March 2003

Interdisciplinary Discussion

76 Surgical Treatment of Obesity

Husemann, B. (Düsseldorf) (Discussion Leader)

Original Articles

81 Vascular Anatomy of the Front Abdominal Wall: A Contribution to Avoid Vessel Injuries during Laparoscopic Surgery

Balzer, K.M. (Düsseldorf); Witte, H. (Jena); Faustmann, P. (Bochum); Kozianka, J.; Waleczek, H. (Herne)

86 Self-Expanding Metal Stents in the Treatment of Colonic Obstruction

Dell'Abate, P.; Del Rio, P.; Colla, G.; Soliani, P.; Arcuri, M.F.; Ziegler, S.; Sianesi, M. (Parma)

89 Palliative Chemotherapy of Colorectal Cancer - Results from an Inquiry

Manekeller, S.; Lauschke, H.; Tolba, R.; Minor, T. (Bonn); Gallkowski, U. (Neuwied); Hirner, A. (Bonn)

Letter to the Editors

96 Improved Early Detection of Gastric Cancer Classen, M. (Gastro-Liga); Riemann, J.F. (Lebensblicke); Schmiegel, W.-H. (DGVS)

99 Book Reviews

101 Meetings and Conferences

2 Imprint

103 Guidelines for Authors

\section{KARGER}

(C) 2003 S. Karger GmbH, Freiburg

Fax +497614520714 